\title{
Frequency Regulation Strategy of Wind Farm Based on Hierarchical
}

\section{Architecture}

\section{Xue Yingcheng ;}

Yancheng Institute Of Technology, Yancheng, Jiangsu 224001, china

Key words: wind farms; frequency control; wind turbine generators;

\begin{abstract}
Based on the analysis of the frequency modulation capability of conventional wind turbines and wind turbines, this paper proposes a new type of frequency control strategy for wind farms, which is based on the layered architecture. By means of reserve control, it can provide fast active power support in the first few seconds, help hydroelectric power-based systems reduce the initial frequency drop, and provide additional active power support in the long term under a certain wind speed. Simulation results show that Wind Farms can participate in frequency Control like Conventional Power Plants to a certain extent.
\end{abstract}

\section{Production}

With the increasing of wind power generation in the power system, the frequency characteristic of the system may be changed. The traditional generator sets are equipped with a frequency modulation control and can contribute to the inertia effect of the system. But in the current wind turbines, most of them require the wind turbine to output the maximum power, and there is no frequency control mechanism. In addition, the current mainstream variable speed wind turbine, due to the use of power electronic conversion equipment, so that it is decoupled from the system frequency, and cannot contribute to the inertia effect of the system; therefore, the current characteristics of the wind turbine system will worsen the system frequency response, so we need to further study the frequency control characteristics of the wind generator, and develop a practical and effective frequency controller for the wind generator.

In order to improve the frequency of the system, more and more power companies require wind power to participate in frequency control, provide ancillary services, at least as a conventional power plant to provide ancillary services or better. The paper [1-2] proposes to modify the control system of variable speed wind turbines, introduce inertia, use the rotational kinetic energy stored in the turbine blades to provide short-term active power support, but the proposed control method cannot provide continuous and stable additional active support. The control strategy proposed in the paper [3] simulates the Droop control of conventional synchronous generators, which provides long-term active power support and participates in frequency control, but the proposed control method does not take into account the FM capability of the wind turbine in different operating conditions. In the paper [4], it is considered that the response of the current controller can be slow in order to use the turbine blades inertia of doubly-fed generators. The paper [5-6] combines the frequency control strategy with the inertial control, but the proposed control method does not consider the standby power and the natural recovery process of the rotor.

Based on the analysis of the frequency modulation ability of the traditional generator set and the wind turbine, new wind farm control system with the whole wind farm as an object is proposed, which can support the active power in a few seconds, and provide additional active power in a 
certain wind speed. In addition, in order to avoid variable speed wind power generation (VSWT) with the rapid growth of output power and the adverse effect on the conventional generator response, a coordinated control strategy between wind turbines and conventional generators is proposed.

\section{Comparison of the output characteristics of conventional and wind power generation}

When system load increases (5 load disturbances), wind power generation with conventional hydroelectric power plants, power generation output power waveform as shown in Figure 1 .

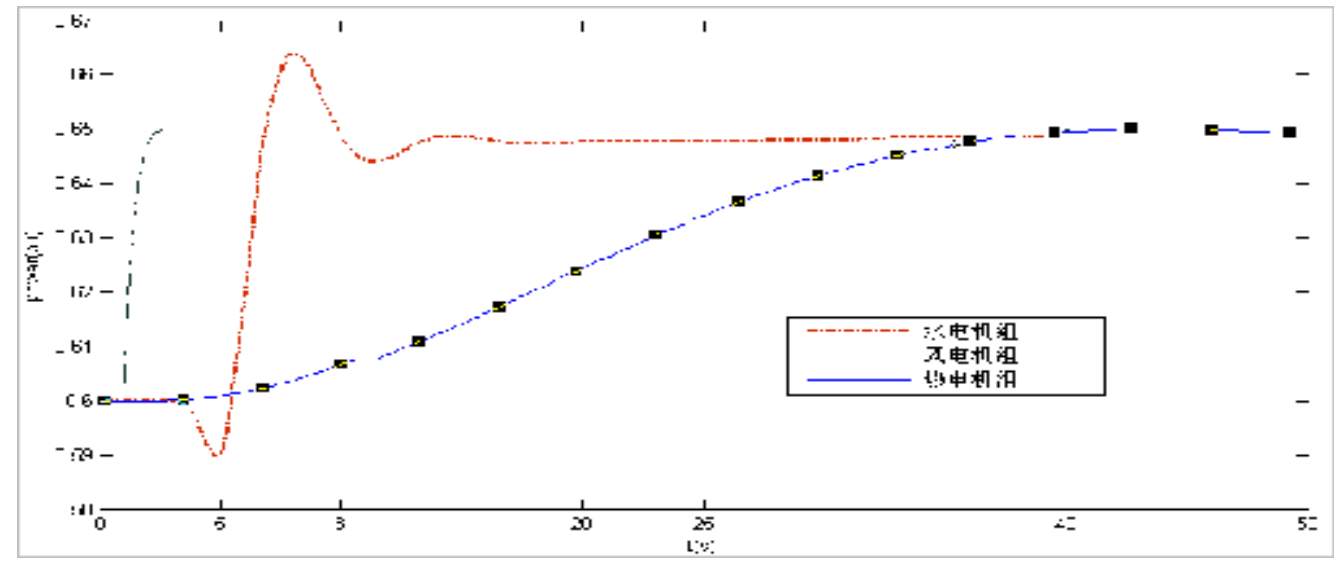

Fig.1 Comparison of wind turbine generators and convention units output response

From the curve in Figure 1, we can see that the response time of different types of power plants is different. The response time and stability time of hydropower and thermal power units are longer than those of wind turbines.

In addition, thermal power, hydropower units due to the governor action to increase the input of the prime mover, can provide continuous additional active support, but the response has a certain delay, and the turbine's initial output power waveform subsidence, while wind power units for frequency changes can make a rapid response, can help hydropower based power system to stop the initial frequency decline.

\section{wind electric field frequency control scheme}

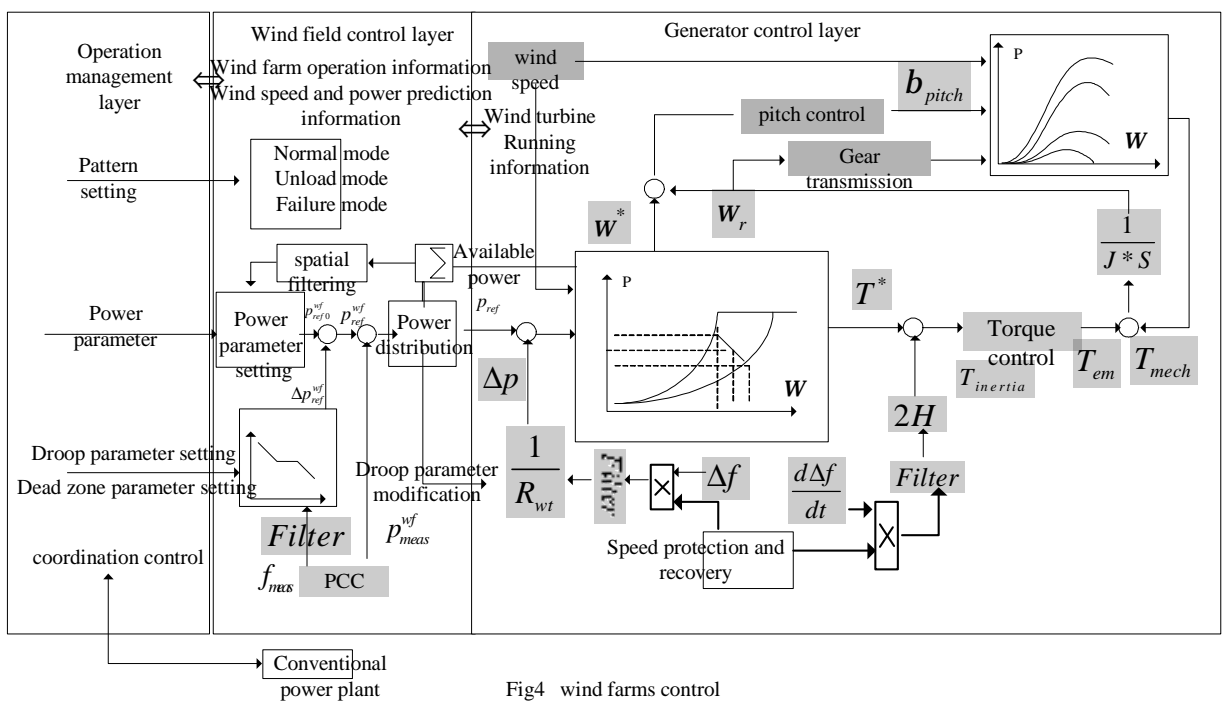

Through the above analysis of the output power of wind turbines, the power characteristics of wind 
turbines are different from those of conventional units. The power characteristics of wind turbines are fast and transient, and the conventional units have time delay and continuity.

Both of them need coordinated control, and each of them has their own advantages. Considering the spatial distribution characteristics of wind farms and the need for Unloading operation for longer term frequency control, a wind farm frequency control scheme is shown in Figure 4.

The new frequency control system is based on hierarchical architecture and operation management layer to supervise the whole wind farm behavior. It determines the control layer mode state, power parameters of wind farms, and coordinates the frequency control between wind farms and conventional power plants. The amount of electricity generated by each wind turbine, estimated available power, wind speed and other information is sent to the control layer of the wind farm. The control layer of the wind farm measures the frequency of the common connection point. According to the droop characteristic curve of the wind farm, when the system frequency changes, the active power output of the entire wind farm is increased or decreased, thus making the wind farm participate in the system frequency control like a conventional power plant. The control layer of the wind farm controls the whole wind farm power generation, sending reference values to each wind farm's local controller. The local control layer can be responsible for each single wind generator control and ensure that the transmitted reference power level is reached.

The control layer of the wind farm

The control layer of the wind farm centrally controls the active and reactive power of the whole wind farm, so that the wind farm can also participate in the control of the power grid as well as the traditional power station. The control system of wind farms usually includes power reference block, wind farm controller itself and a dispatching (allocation) control block. The delay of communication signals between wind farms is ignored.

The control layer of the wind farm can be operated in the maximum power mode, the reserve control mode, and the failure mode according to the requirements of the system operators. In the normal maximum power mode, the wind farm can not increase the power input of prime mover, and it can use the rotational kinetic energy stored in turbine blades to provide short term active power support under the rated power. In the reserve control mode, a part of the active output is reduced by controlling the pitch angle or adjusting the power - speed optimal curve, and this part of the active output is reserved as the reserve power. Whenever the large frequency deviation is detected, additional power reference is added to the set point, which in turn will further adjust the pitch angle to get more power from the wind. It can support fast and active power in a few seconds. It can help a hydropower based system to reduce initial frequency and provide extra active power for a long time. In communication failure mode, each wind turbine participates in frequency control according to its own frequency controller, and the coordinated control between wind turbines and conventional power plants is out of action. Automatic frequency control in the control layer of wind farms is designed to avoid the conflict between power control and frequency control of the wind turbine, and to reduce the adverse effects of excessive operation of wind turbines. The control layer of the wind farm is mainly responsible for the situation of the large frequency change and slow speed of the frequency change. The control layer of the wind turbine is mainly responsible for the situation of small frequency change and fast speed change.

The dispatch and distribution control module distributes the output power of the whole wind farm, and converts the output power to the power reference signal of the single wind turbine. According to the requirements of the system operator and the operation state of the wind farm, the control level of the wind farm calculates the reference signal of each wind turbine, determines the slope parameters of each wind turbine, and adjusts the power output speed, determines the need for 
reserve capacity control (balance control, power control, delta slope restrictions, automatic frequency control), the initial distribution is generally in proportion to their own capacities to adjust the amount of power.

In reserve control mode, the wind farms must allocate a reserve power rationally according to their own operation information ay, so as to ensure that the system operators get the ideal reserves. Assuming that the total generation margin should not be less than $10 \%$ of the available power, according to the predicted wind speed at a certain time in the future, it is estimated that the average output of the wind farm is $\mathrm{P}_{\text {avg }}$, so the wind farm can provide a reserve $\mathrm{P}_{\mathrm{WF}}$ :

$$
P_{W F}=P_{a v g} \times 10 \% M W \text {. }
$$

Because the power extraction curve of the wind turbine ((WT) can be related to the wind speed, the higher the wind speed, the greater the power generation allowance. Therefore, the wind farm reserve $\mathrm{P}_{\mathrm{WF}}$ is related to the wind speed of each wind turbine, and the distribution factor DF (VW) is set as:

$$
D F_{W T}\left(V_{\omega}\right)=\frac{W\left(V_{\omega}\right) \times n\left(V_{\omega}\right)}{\sum\left[W\left(V_{\omega}\right) \times n\left(V_{\omega}\right)\right]} \times \frac{1}{n\left(V_{\omega}\right)}
$$

Among them, $W\left(V_{\omega}\right)$ is the weight factor of each level of wind speed. The higher the wind speed is, the greater the 2 weighted value is. The $n\left(V_{\omega}\right)$ is the number of wind turbines running at $V_{\omega}$ wind speed. The denominator of (2) includes all the wind turbines that can be used for unloading operation. The power generation allowance for each wind turbine at the wind speed is:

$$
P_{W T}\left(V_{\omega}\right)=P_{W F} \times D F_{W T}\left(V_{\omega}\right) .
$$

In order to ensure that the speed of the wind turbine is higher, the reaction of the droop is faster. According to the distribution coefficient, the droop coefficient $\mathrm{R}_{\mathrm{WF}}$ of each wind turbine is:

$$
\frac{1}{R_{W T}\left(V_{\omega}\right)}=\frac{1}{R_{W F}} \times D F_{W T}\left(V_{\omega}\right)
$$

All three blocks mentioned in the control layer of wind farm (power benchmark setting block, main controller module, dispatching block and output power slope) have upper limit regulatory signals.

\section{2 wind turbine control layer}

Wind turbine control must be able to adjust the power of the wind generator, and the reference value is calculated by the control layer of the wind field according to the requirements specified by the system operator.

Each wind generator sends the state information back to the control layer of the wind farm in each control cycle, and the power and wind speed should be filtered to avoid unnecessary fluctuations.According to the input wind speed and the maximum and minimum power curve of wind turbines, each wind turbine obtains the adjustable power range $\mathrm{P}_{\text {margin }}$ of the wind turbine, which is also sent to the control layer of the wind farm. The wind power control layer sets the power $\mathrm{P}_{\text {set }}$ of each wind turbine by the power distribution control module, according to the $\mathrm{P}_{\text {margin }}$, wind speed and other information. through the power control module of each wind turbine, coupled with frequency droop control, adjust the amount of power generated by the $\Delta \mathrm{P}$, can get the output power of each wind turbine reference value $\mathrm{P}_{\text {operate }}$.

The reference speed wref and the output torque of wind turbine Tem* can be obtained from the 
wind turbine output power reference $\mathrm{P}_{\text {operate }}$ and the maximum and minimum power curve of the wind turbine.

The sum of the output power Tem* and the inertia torque Tinertia of the wind turbine is the reference torque of the generator DFIG. In order to minimize the influence on the mechanical drive chain, a first-order high pass filter is added to reduce the power (torque) change rate..

Speed Protection Recovery module, in order to prevent FM and rotor speed below the minimum value of $\omega_{\text {min }}$, resulting in speed recovery difficulties, affecting the stability of the system. When the speed is lower than $\omega_{\min }(\mathrm{n}$ is generally set to 0.7$)$, the speed protection system will set the $\Delta P F$ to 0 , no longer participate in the system frequency control; For large wind farm units, all units at the same time into the speed recovery mode, the supply of active power at the same time reduce, may cause the system frequency of two times fall . Therefore, in order to reduce the speed recovery function to weaken the active support, the sequential recovery method can be taken, all the units in a certain delay time in turn into the speed recovery process.

The operations management layer

Operation management layer supervises the wind farm behavior, which determines the control layer mode state of the wind farm, power parameters, and coordination with conventional power plants. In addition, the rapid increase in output power of variable speed wind power slows down the response of conventional generators to some extent. To avoid such adverse effects, it is suggested that coordination between non-conventional generators and a set of control laws for selected conventional generators.

The coordinated control module of conventional generators is mainly concerned with giving full play to the rapidity of wind turbines and the continuity of conventional units, so as to provide more effective frequency support for the system.

Output power coordination of generator

Coordinated generation control is integrated into automatic gain control to manage power regulation, control power balance and allocate imbalance among selected units in an economic way. According to the operation time constant of different generators, the capacity and capacity of the following load frequency controller (LFC) and the contribution to the two times frequency regulation control, the participation coefficient Kci is set for each conventional generator and the wind turbine.

$\sum_{i} K_{C i}=1$

Set $P_{n}^{*}$ for the total extra injection power, the extra power for each generator is:

$$
P_{C i}=K_{C i} P_{n}^{*}
$$

The rapidity and transients of the wind turbine generator units should be coordinated with the delay and continuity of the conventional units. The coordinated control and coordinated generation control models are shown in Figure 5. 


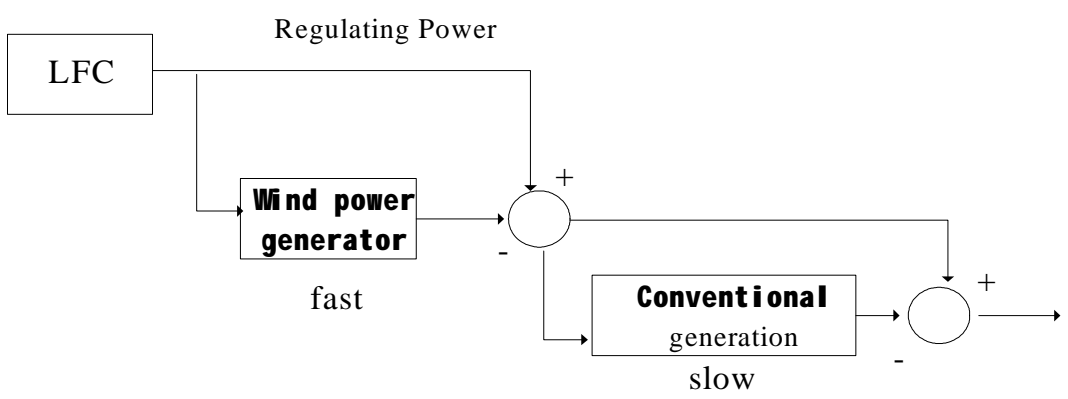

Fig.5 The coordinated generation control

\section{Reserve capacity coordination}

As the maximum power fluctuates with the wind, it may lead to different deloaded conditions, and the range of unload allowance depends on the wind speed. It is assumed that the system load damping is Dest, one time reserve is $\mathrm{P}_{\mathrm{RSYS}}$, and in the 50 hertz system, the calculation is as follows:

$P_{\text {Rsys }}=\Delta P_{G \max }-D_{\text {est }} \times\left(50-F_{\text {safe }}\right) M W$.

$\Delta p_{\text {Rsys }}$ is the most credible single emergency reserve in the system, $\mathrm{F}_{\text {safe }}$ is an ideal security frequency. Combined with the estimated $\mathrm{P}_{\mathrm{rsys}}$, the traditional power plant reserves $\mathrm{P}_{\mathrm{RC}}$ are:

$P_{R c} \geq\left(P_{R s y s}-P_{W F}\right) M W$.

The equal sign indicates the minimum reserve requirement of the traditional unit to ensure that the system frequency is not less than $\mathrm{F}_{\text {safe. }}$.

\section{Simulation Research}

simulation system model

The operating management simulation of the power system consists of a conventional synchronous generating unit (total $9 \mathrm{MW}, \mathrm{pf}=0.8, \mathrm{~h}=1.07 \mathrm{~s}$ ) and a total installed capacity of $9 \mathrm{MW}$ wind farms, composed of $1.5^{*} 6 \mathrm{MW}$ doubly-fed wind turbines, generators and loads connected to the $35 \mathrm{kV}$ bus, as shown in Figure 6 , The synchronous generator includes the six-order model of the synchronous generator, the excitation system, the primary motivation and the Speed regulation system model [9]. The two-mass model is adopted for the mechanical axis of wind turbine, doubly-fed generator is equipped with decoupling P, Q Power controller, stator magnetic field orientation, D and Q-axis rotor current respectively control the stator active and reactive power [10], and run in the unit wattage factor.Assume the wind speed is constant (unless otherwise specified) and consider the effect of the basic aerodynamics.

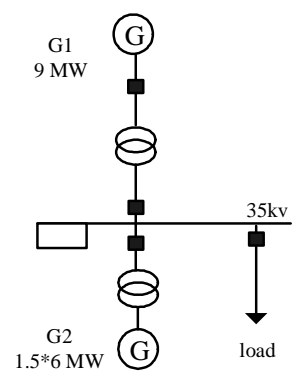

Fig.6.Power system model 


\section{Simulation and analysis of reserve operation and reserve capacity}

Reserve operation simulation results as shown in Figure 7, Figure 7 (a) for wind speed, 7 (b) is the active power output under the maximum wind energy extraction mode (MDP).Figure 7 (c) is the active power output under the constant reference power reserve operating mode at the same wind speed.The reference power is set to $0.9 \mathrm{pu}$. Figure 7 (d) is the power generation allowance. As can be seen from Figure 7 (d), the generation margin exists only if the maximum power extracted from the wind is greater than the reference power. The reserve obtained remains unchanged at $0.1 \mathrm{pu}$ when the wind speed is higher than the rated wind speed. When the maximum power is between $0.9 \mathrm{pu}$ and $1.0 \mathrm{pu}$, the reserve varies with the wind speed and is zero when the maximum power is less than $0.9 \mathrm{pu}$.

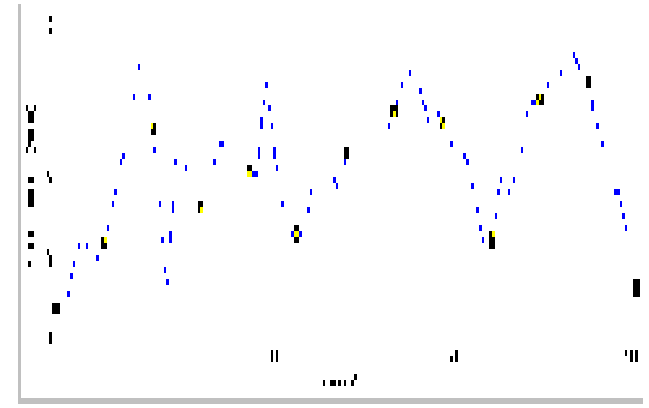

Fig.7（a） wind $\operatorname{speed}(\mathrm{m} / \mathrm{s})$

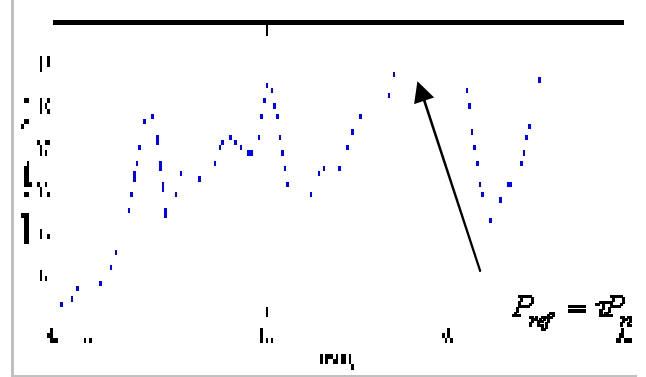

Fig.7 (c) constant power deload operation mode

Fig.7 (d) constant power deload operation mode reserve

The effect of droop control on the frequency response of a doubly fed generator

The load of $15 \%$ increases in $t=4$ seconds, and the system frequency decreases until the automatic generation increases the output power and frequency recovery. The frequency response of different droop coefficients $\mathrm{R}=12 \%$ and $\mathrm{R}=10 \%$ is shown in Figure 7 (a). The droop coefficient is reduced, the rate of frequency descent is almost unchanged, the smaller the minimum frequency, the smaller $\mathrm{R}$, and the greater the active power of the wind power generation, the smaller the frequency offset, which improves the frequency response and the robustness of the whole system.

But if $\mathrm{R}$ is too small, the mechanical power of blades will be reduced too much. If the frequency is not recovered, the electromagnetic torque may continue to be greater than the aerodynamic torque, causing the wind turbine to stop, as shown in Figure 7 (b). Therefore, in the power system with large frequency variation, such as the microgrid island system, the stator output power should be carefully controlled (without causing the wind turbine to stop), so as to prevent the instability of the wind turbine. 

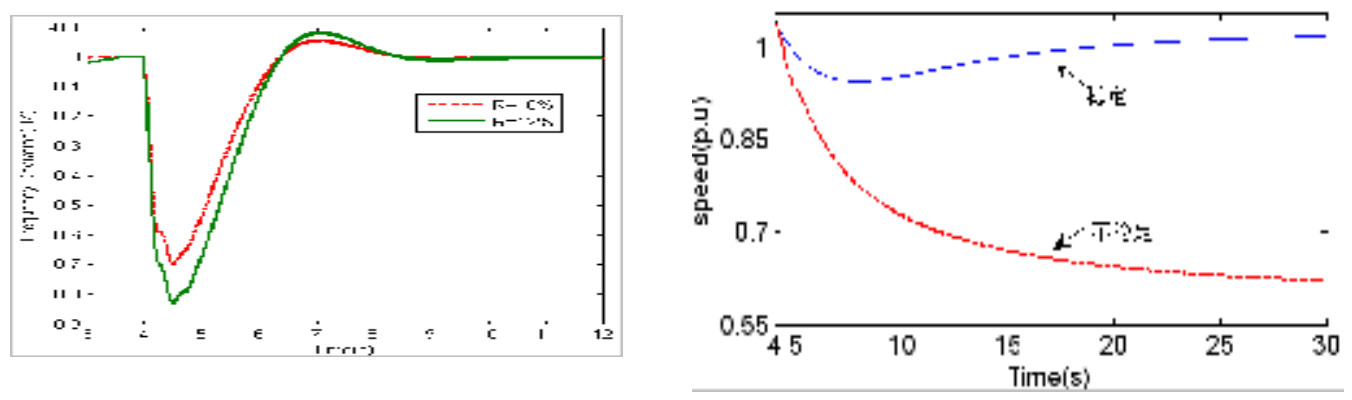

Fig. 9 Influence of controller Parameters on inertial response.

4.3 coordinated control simulation research

Figure 10 is the response curve of the system frequency and the active power of the conventional unit after increasing the coordination control link. When the coordination signal PCi is considered, the frequency waveform of the system is greatly improved and the minimum frequency is smaller. Through the coordinated control of the wind turbine and the conventional unit, the conventional generator can be more effectively involved in the system frequency regulation, which is beneficial to the support of the system frequency.
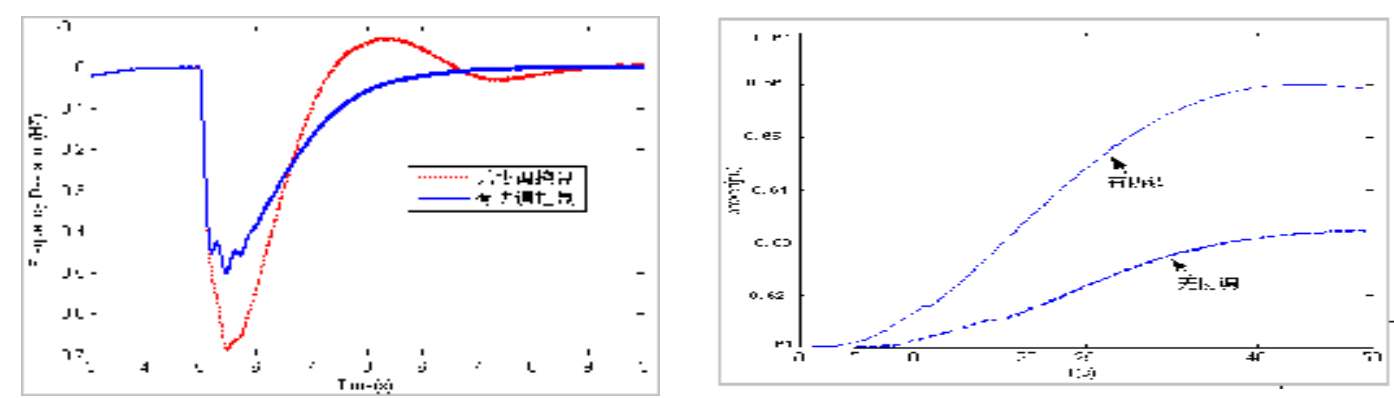

Fig.10.Frequency and active power responses of convention units with coordination control

\section{Conclusion}

Based on the analysis of the frequency modulation ability of conventional units and wind turbines, a new wind farm control system with the whole wind farm as object is proposed, which makes the wind farm participate in the system frequency control as a conventional power plant. The system is based on hierarchical architecture. By reserve control, it can provide fast active power support in the first few seconds, and it can help a hydropower based system to reduce initial frequency and provide extra active power for a long time. In addition, the output power of the variable speed wind power generation increases rapidly and slows down the response of the conventional generator to a certain extent. In order to avoid such adverse effects, a coordinated control strategy between the unconventional generator and the conventional generator is proposed.

Through the simulation and calculation of typical megawatt variable speed wind power generation, it is shown that in the next 10 seconds, variable speed wind power can easily provide additional 0.1 times active power support, fast response to frequency changes, and provide effective power support. Through coordinated control of wind turbines and conventional generating units, conventional generators can participate more effectively in system frequency regulation, and support system frequency. It is proved that wind farms based on variable speed doubly fed generators can participate in the frequency control of the system to a certain extent.

\section{Reference}


[1]J.Enanayake and N.Jenkins,Comparison of the response of doubly fed and fixed speed induction generator wind turbines to changes in system frequency, IEEE Trans.Energy Convers.,vol.19,no.4,pp.800-802,Dec.2004.

[2] J. Morren, S. W. H. de Haan, W. L. Kling, and J. A. Ferreira, Wind turbines emulating inertia and supporting primary frequency control,IEEE Trans. Power. Syst., vol. 21, no. 1, pp. 433-434, Feb. 2006.

[3] KEUNG P K, LI P, BANAKAR H, et al. Kinetic energy wind-turbine generators for system frequency support. IEEETrans on Power Systems, 2009, 24(1): 279-287.

[4] MAURICIO J M, MARANO A, MEZ-EXP SITO A G,et al. Frequency regulation contribution through variable-speed wind energy conversion systems. IEEE Trans on PoweSystems, 2009, 24(1): 173-179.

[5]G.Ramtharan et al., Frequency support from doubly fed induction generator wind turbines, IET Renew.Power Gen.,vol.1,no.1,pp.3-9,Mar.2007.

[6]GUAN Hongliang, CHI Yongning, WANG Weisheng, et al. Simulation on frequency control of doubly fed induction generator based wind turbine. Automation of Electric Powe Systems, 2007, 31(7): 61-65.

[7] R. G. de Almeida and J. A. Pecas Lopes, "Participation of doubly fed induction wind generators in system frequency regulation," IEEETrans. Power Syst., vol. 22, no. 3, pp. 944-950, Aug. 2007.

[8] R. G. de Almeida, E. D. Castronuovo, and J. A. Pecas Lopes, Optimum generation control in wind parks when carrying out system operator requests, IEEE Trans. Power Syst., vol. 21, no. 2, pp. 718-725, May2006.

[9]Chemmangot V. Nayar,, Mochamad Ashari, W. W. L. Keerthipala: "A grid-interactive photovoltaic uninterruptible power supply sys-tem using battery storage and a back up diesel generator", IEEE Transactions on Energy Conversion, Vol. 15, No. 3, September 2000.

[10] M. Karari, W. Rosehart and O.P. Malik, "Comprehensive control strategy for a variable speedcage machine wind generator unit", IEEE Transactionson Energy Conversion, vol. 20, No. 2, pp. 415-423,June 2005. 